\title{
DIFFUSION OF SPHERES IN A CONCENTRATED SUSPENSION: RESUMMATION OF MANY-BODY HYDRODYNAMIC INTERACTIONS
}

\author{
C.W.J. BEENAKKER and P. MAZUR \\ Instituut-Lorentz, Rijksuniversiteit Leiden, Nieuwsteeg 18,2311 SB Leiden, The Netherlands
}

Received 5 July 1983

\begin{abstract}
We evaluate the wavevector dependent (short-time) diffusion coefficient $D(k)$ for spherical particles in suspension. Our analysis is valid up to high concentrations and fully takes into account the many-body hydrodynamic interactions between an arbitrary number of spheres. By resumming moreover a certain class of correlations, we obtain results which agree well with available experimental data for the small and large wavevector limits of $D(k)$.
\end{abstract}

\begin{abstract}
1. Recently [1] we evaluated the concentration dependence of the (short-time) self-diffusion coefficient for spherical particles suspended in a fluid. This quantity, denoted by $D_{\mathrm{s}}$, is the large- $k$ limit of a wavevector dependent diffusion coefficient $D(k)$, which describes the initial decay of the dynamic structurefactor $S(k, t)$ measured by inelastic light or neutron scattering [2]. In our analysis [1] we resummed the hydrodynamic interactions between an arbitrary number of spheres. The importance of these many-body interactions has been demonstrated both theoretically [3] and experimentally [4]. By including at most two-point correlations between the spheres, we obtained in ref. [1] a reasonable agreement with experimental results for $D_{\mathrm{s}}[4]$, for volume fractions $\varphi \lesssim 0.30$. At higher concentrations the calculated values were too large, indicating the importance of higher order correlations.

The extension to ref. [1] presented here is twofold: (i) we extend the formalism to diffusion at arbitrary (non-zero) values of the wavevector; (ii) we resum to all orders the contributions due to a certain class of correlations, the so-called ring-correlations, thereby obtaining results for $D_{\mathrm{s}}$ which agree with the experimental data up to the highest volume fractions.
\end{abstract}

2. The (short-time) diffusion coefficient $D(k)$ is given by [2]

$$
\begin{aligned}
& k^{2} S(k) D(k)=k_{\mathrm{B}} T N^{-1} \\
& \quad \times \sum_{i, j=1}^{N} k \boldsymbol{k}:\left\langle\boldsymbol{\mu}_{i j} \exp \left[\mathrm{i} \boldsymbol{k} \cdot\left(\boldsymbol{R}_{j}-\boldsymbol{R}_{i}\right)\right]\right\rangle,
\end{aligned}
$$

in terms of an average of the mobility tensor $\boldsymbol{\mu}_{i j}$ of spheres $i$ and $j$, which have positions $R_{i}$ and $R_{j}$ respectively. The mobilities depend on the whole configuration of the $N$ spheres and may be calculated from the linear Stokes equation [5]. Also, $S(k)$ is the static structurefactor and $k_{\mathrm{B}}$ and $T$ denote Boltzmann's constant and the temperature respectively.

Adopting the notation used in ref. [1], we may write $^{\ddagger 1}$

$$
\begin{aligned}
& S(k) D(k) / D_{0}-1 \\
& \quad=-k^{-2} N^{-1} k k: P\left\langle\left[n A(1-n Q A)^{-1} n\right](k \mid k)\right\rangle P .
\end{aligned}
$$

In eq. (2) an average is taken of the $k, k$ element of the integral operator between braces. The propagator $A$ is a matrix of which the elements characterize a hydrodynamic interaction between two induced-force multipoles. The microscopic numberdensity of the spheres is given by $n$. In $r$ representation, the elements of $A$ are convolution operators and $n$ is a diagonal operator. The object $P=1-Q$ projects out the first multipole moment of an induced force. The Stokes-Einstein value

${ }^{\ddagger 1}$ The operator $A$ used in eq. (2) corresponds to $\mathcal{B}^{-1} \mathscr{A}$ in ref. [1]. 
of the diffusion coefficient is denoted by $D_{0}$. In the limit $k \rightarrow \infty$, eq. (2) reduces to the expression for $D_{\mathrm{s}}$ given in ref. [1].

3. The expression between braces in eq. (2) may be expanded in powers of the density fluctuations $\delta n \equiv$ $n-n_{0}$, where $n_{0}$ is the average numberdensity of the spheres. In ref. [1] we evaluated $D_{\mathrm{s}}$ to second order in this so-called fluctuation expansion. There is however a certain class of contributions due to correlations which may be resummed to all orders. To this end we define for arbitrary $\gamma_{0}$ a renormalized propagator $A_{\gamma_{0}}$ by

$A_{\gamma_{0}} \equiv A\left(1-\gamma_{0} Q A\right)^{-1}$.

We choose $\gamma_{0}$ to be a function of the concentration $n_{0}$,

$\gamma_{0}\left[1-Q A_{\gamma_{0}}(r=0)\right]=n_{0}$,

where $A_{\gamma_{0}}(r)$ is the kernel of the operator $A_{\gamma_{0}}$ defined above. One may now prove the identity

$A(1-n Q A)^{-1} n=A_{\gamma_{0}}\left(1-\delta \gamma Q{\stackrel{\circ}{\gamma_{0}}}\right)^{-1} \gamma$,

where $\gamma \equiv \gamma_{0}+\delta \gamma \equiv n \gamma_{0} / n_{0}$ is a renormalized vertex and $\AA_{\gamma_{0}}$ is a cut-out propagator with kernel

$\AA_{\gamma_{0}}(r)=A_{\gamma_{0}}(r)$ for $r \neq 0, \quad \AA_{\gamma_{0}}(r=0)=0$.

In ref. [1] we defined a renormalized propagator $A_{n_{0}}$ according to eq. (3), with $\gamma_{0}$ equal to $n_{0}$, and proved the identity

$A(1-n Q A)^{-1} n=A_{n_{0}}\left(1-\delta n Q A_{n_{0}}\right)^{-1} n$,

which did not contain a renormalized vertex, nor a cutout propagator. Both expressions (5) and (7) are equivalent. However, the zeroth order term in the $\delta \gamma$-expansion differs from the corresponding term in the $\delta n$-expansion: the latter contains the full hydrodynamic interactions between an arbitrary number of spheres in the absence of correlations, while the former moreover contains a class of self-correlations. In a diagrammatic representation this class corresponds to ringdiagrams. Through formulae (3) and (4) the resummation of these diagrams, is performed algebraically. Furthermore, the contributions of order $(\delta \gamma)^{2}$ in the $\delta \gamma$-expansion are much smaller than those of order $(\delta n)^{2}$ in the fluctuation expansion described in ref. [1]. This indicates that the former expansion converges faster than the latter one. We remark that to linear order in the density $\gamma_{0}$ equals $n_{0}$ and both fluctuation expansions are identical.

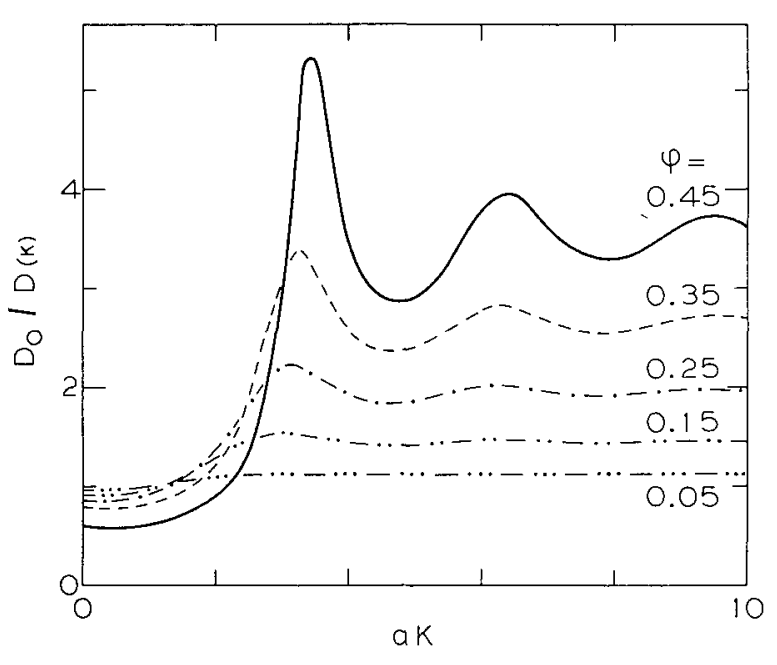

Fig. 1. $D_{0} / D(k)$ as a function of the wavevector $k$ times the particle radius $a$, for five values of the volume fraction $\varphi$.

4. We have evaluated $D(k)$ given by eq. (2) up to and including terms of second order in $\delta \gamma$, using eq. (5). The pair distributionfunction (necessary for the calculation of $S(k)$ and $\left.\left\langle(\delta \gamma)^{2}\right\rangle\right)$ was approximated by the solution of the Percus-Yevick equation. The resulting wavevector dependence of $D_{0} / D(k)$ is plotted in fig. 1, for five values of the volume fraction $\varphi$ [note that in the absence of hydrodynamic interactions $D_{0} / D(k)$ equals the

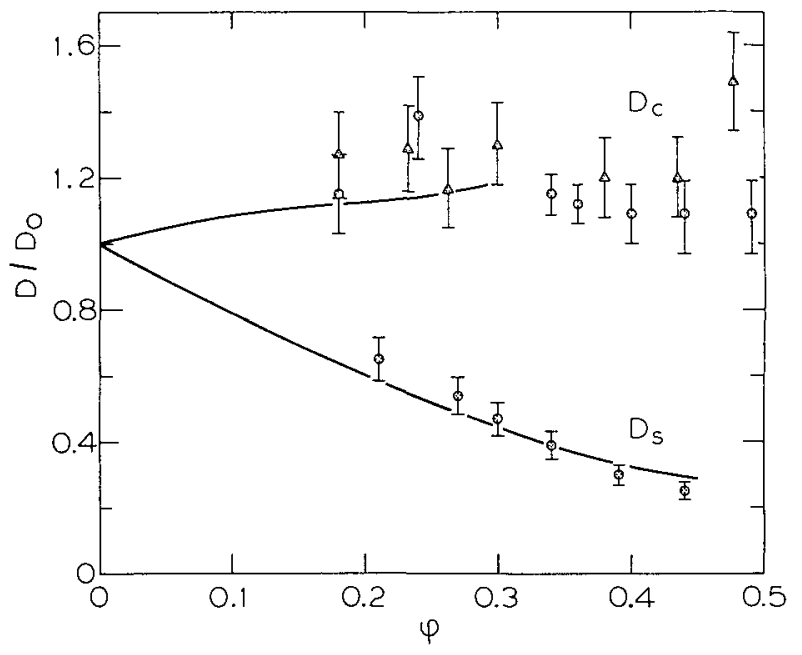

Fig. 2. $D_{\mathrm{S}} / D_{0}$ and $D_{\mathrm{C}} / D_{0}$ as a function of the volume fraction $\varphi$. The solid lines give the results of our calculations. Experimental data for $D_{\mathrm{s}}$ (shown by dots) are from ref. [4]; for $D_{\mathrm{c}}$, dots are from ref. [6] and triangles from ref. [7]. 
structurefactor $S(k)]$. In fig. 2 we have plotted the concentration dependence of the two limits

$D_{\mathrm{s}}=\lim _{k \rightarrow \infty} D(k)$ and $D_{\mathrm{c}}=\lim _{k \rightarrow \infty} D(k)$,

together with experimental results. The theoretical values for the self-diffusion coefficient $D_{\mathrm{s}}$ are given for $\varphi \leqslant 0.45$, while the values for the collective diffusion coefficient $D_{\mathrm{c}}$ are only shown for $\varphi \leqslant 0.30$. At higher concentrations the calculated values for $D_{\mathrm{c}}$ become less and less reliable due to cancellations.

For $D_{\mathrm{s}}$ agreement with the experiments of Pusey and van Megen [4] is obtained, up to the highest (experimental) volume fractions. The experimental data $[6,7]$ for $D_{c}$ indicate that this quantity is rather insensitive to changes in the concentration over a large range of volume fractions; a remarkable result, which is confirmed by our calculations.

Details of this work will be given elsewhere.
This investigation was performed as part of the research programme of the "Stichting voor Fundamenteel Onderzoek der Materie" (F.O.M.), with financial support from the "Nederlandse organisatie voor Zuiver Wetenschappelijk Onderzoek" (Z.W.O.).

References

[1] C.W.J. Beenakker and P. Mazur, Physica A (1983), to be published.

[2] P.N. Pusey and R.J.A. Tough, J. Phys. A15 (1982) 1291.

[3] C.W.J. Beenakker and Mazur, Phys. Lett. 91A (1982) 290.

[4] P.N. Pusey and W. van Megen, J. Phys. (Paris) 44 (1983) 285.

[5] P. Mazur and W. van Saarloos, Physica 115A (1982) 21.

[6] M.M. Kops-Werkhoven and H.M. Fijnaut, J. Chem. Phys. 77 (1982) 2242.

[7] D.J. Cebula, R.H. Ottewill, J. Ralston and P.N. Pusey, J. Chem. Soc. Faraday Trans. 177 (1981) 2585. 